\title{
The Second Messenger cAMP Elicits Eating by an Anatomically Specific Action in the Perifornical Hypothalamus
}

\author{
Elizabeth R. Gillard,, ${ }^{1}$ Arshad M. Khan, ${ }^{2}$ Rickinder S. Grewal, ${ }^{1}$ Bara Mouradi, ${ }^{1}$ Stefany D. Wolfsohn, ${ }^{1}$ and \\ B. Glenn Stanley ${ }^{1}$ \\ ${ }^{1}$ Departments of Neuroscience and Psychology and ${ }^{2}$ Division of Biomedical Sciences and Department of Neuroscience, \\ University of California at Riverside, Riverside, California 92521
}

We have previously shown that a membrane-permeant analog of cAMP, 8-bromo-cAMP (8-br-cAMP), elicits a vigorous eating response when microinjected into the perifornical hypothalamus (PFH) or lateral hypothalamus (LH) of satiated rats, suggesting that increases in cAMP in these areas may be important in the neural control of eating. To determine the locus of this effect, we compared the ability of 8-br-cAMP (1-100 nmol/0.3 $\mu l)$ to elicit eating after microinjection into the $\mathrm{PFH}, \mathrm{LH}$, or the following bracketing areas: the anterior and posterior $\mathrm{LH}$, paraventricular nucleus of the hypothalamus, thalamus, and amygdala. 8-br-cAMP at $50 \mathrm{nmol}$ elicited eating ( $\geq 3.4 \mathrm{gm}$ in $2 \mathrm{hr}$ ) exclusively in the PFH and LH. At 100 nmol, 8-br-cAMP elicited

Several lines of evidence suggest that the lateral hypothalamus (LH) and adjacent perifornical hypothalamus (PFH) are intimately involved in the neural control of food intake. Specifically, neurotoxic and electrical lesions of the $\mathrm{LH}$ and $\mathrm{PFH}$ disrupt eating (Anand and Brobeck, 1951; Winn et al., 1984), whereas electrical stimulation in these areas elicits eating in satiated animals (Murzi et al., 1991). Similarly, neurochemical stimulation of LH neurons by glutamate and its receptor agonists elicits intense eating in satiated rats, and glutamate and its receptors have been suggested to play a physiological role in the control of eating and body weight (Stanley et al., 1996). Likewise, the PFH is most sensitive to both the eating-stimulatory effects of neuropeptide Y (Stanley et al., 1993) and the feeding-suppressive effects of catecholamine neurotransmitters, which may participate in the physiological control of eating (Leibowitz and Stanley, 1986). Neurons in the $\mathrm{LH}$ and PFH also respond to internal signals related to the nutritive state of the animal, such as changes in circulating levels of the metabolic fuel glucose (Himmi et al., 1988). More strikingly, LH and PFH neurons respond with changes in firing rate to the sight, smell, and taste of food that both precede and predict feeding in the hungry monkey (Rolls et al., 1986). These neurons also acquire responses to non-food objects paired repeatedly with food, in parallel with the learning of behavioral responses to the objects (Mora et al., 1976).

These data suggest that neuronal elements in the $\mathrm{LH}$ and $\mathrm{PFH}$ are responsive to multiple neurotransmitter inputs relaying information about the internal and external environment, and that

\footnotetext{
Received Aug. 12, 1997; revised Dec. 29, 1997; accepted January 8, 1998.

This study was supported by National Institutes of Health Grant NS24268, Eli Lilly, Sigma $\mathrm{Xi}$, and the University of California, Riverside Department of Neuroscience.

Correspondence should be addressed to Dr. E. R. Gillard, Department of Psychology, University of California at Riverside, Riverside, CA 92521.

Copyright (C) 1998 Society for Neuroscience $0270-6474 / 98 / 182646-07 \$ 05.00 / 0$
}

a larger response in these areas and elicited a smaller, more variable response in the thalamus. We similarly mapped the feeding-stimulatory effects of compounds that increase endogenous cellular cAMP in naive rats. Combined microinjection of matched doses $(300 \mathrm{nmol})$ of 3-isobutyl-1-methylxanthine and 7-deacetyl-7-O-( $N$-methylpiperazino)- $\gamma$-butyryl-forskolin was effective exclusively in the PFH, eliciting an average $2 \mathrm{hr}$ food intake of $8.4 \pm 2.0 \mathrm{gm}$. Collectively, these results suggest that increases in cellular cAMP within a specific brain site, the $\mathrm{PFH}$, may play a role in the neural stimulation of eating.

Key words: perifornical hypothalamus; hypothalamus; cAMP; feeding; second messengers; eating; rat

integration of this information by $\mathrm{LH}$ and $\mathrm{PFH}$ neurons may be important in the neural control of eating. The activity of individual neurons in these areas is modulated in vitro (Cheng et al., 1988) by several neurotransmitters that affect eating, suggesting that individual cells may integrate the effects of a variety of feeding-related neurochemicals. Recent evidence suggests that the cAMP-synthesizing enzyme adenylyl cyclase (AC) may function as a coincidence detector for activation of a variety of metabotropic receptors (Lustig et al., 1993), and we have shown that PFH and LH microinjection of the cAMP analog 8-bromocAMP (8-br-cAMP) or drugs that increase levels of endogenous cAMP elicits a robust eating response in satiated animals (Gillard et al., 1997a). These findings may suggest that cAMP in a population of LH and/or PFH neurons might participate in integrating the feeding-stimulatory and feeding-inhibitory effects of some neurotransmitters. However, because diffusion of centrally injected substances is common (Myers et al., 1971), it is also possible that the eating-stimulatory effects we observed were consequent to diffusion away from the injection site and actions outside of the $\mathrm{LH}$ and $\mathrm{PFH}$. To resolve this issue, in the current study, we compared the ability of 8-br-cAMP and agents that increase cAMP levels to stimulate eating when injected into the $\mathrm{PFH}$ and $\mathrm{LH}$ with their effectiveness when injected into sites bracketing these areas.

Portions of this study have been presented in preliminary form (Gillard et al., 1994, 1997b).

\section{MATERIALS AND METHODS}

Subjects and surgery. A total of 145 adult male Sprague Dawley rats obtained from Charles River Laboratories (Wilmington, MA) were individually housed in a vivarium at $21^{\circ} \mathrm{C}$ with a $12 \mathrm{hr}$ light/dark cycle and maintained on Purina Rat Chow pellets and water ad libitum until 3 d after surgery, when the pellets were removed and permanently replaced with a sweetened milk-mash diet $(46 \%$ Purina Rat Chow 

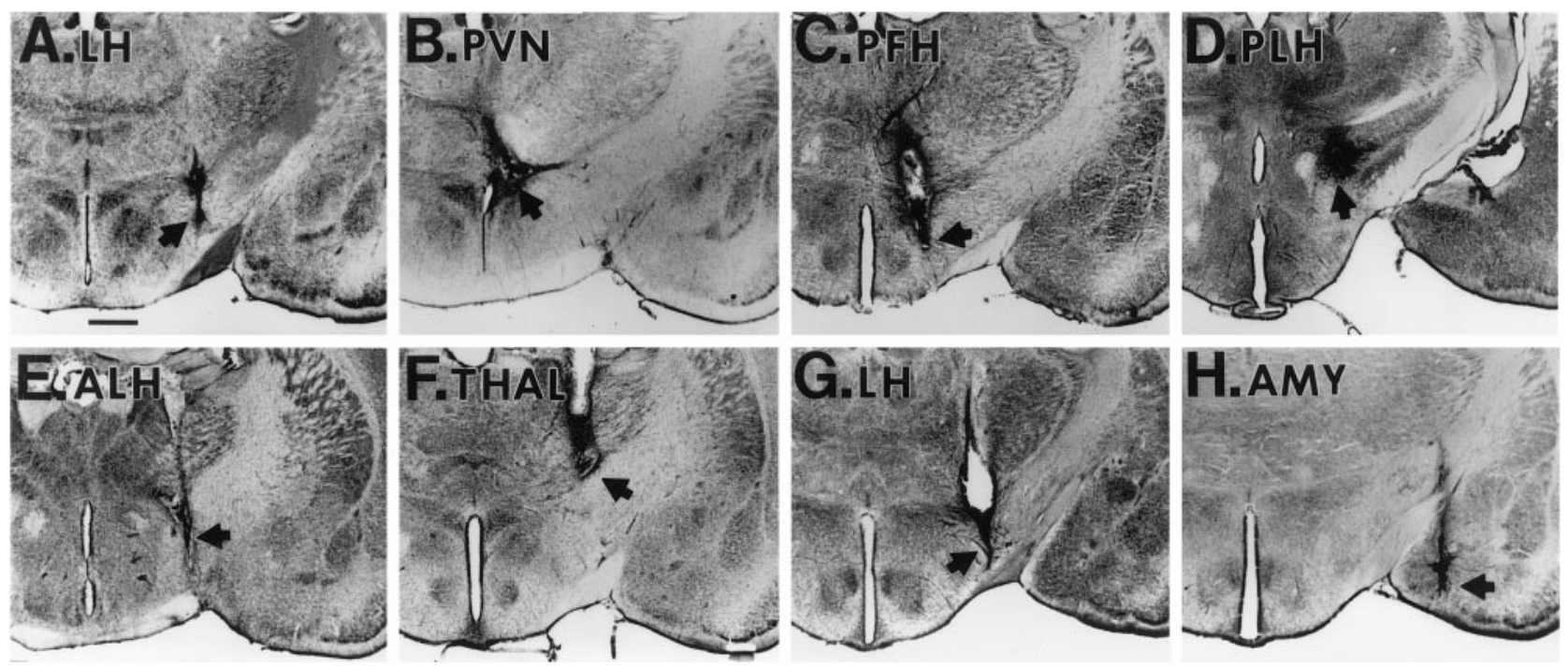

Figure 1. Photomicrographs of coronal sections stained with cresyl violet showing representative injection sites, indicated by arrows, located in $A$, $G$, lateral hypothalamus $(L H) ; B$, paraventricular nucleus of the hypothalamus $(P V N) ; C$, perifornical hypothalamus $(P F H)$; $D$, posterior lateral hypothalamus $(P L H) ; E$, anterior hypothalamus $(A L H) ; F$, thalamus $(T H A L) ; H$, amygdala $(A M Y)$. The top row shows 8-br-cAMP injection sites, and the bottom row shows IBMX-MPB forskolin injection sites. Scale bar, $1 \mathrm{~mm}$.

powder, $37 \%$ sucrose, and 17\% Carnation evaporated milk) to which they had free access for the duration of the experiment.

Animals weighing 350-450 gm were anesthetized by Metofane inhalation and implanted stereotaxically with a chronic unilateral 26 gauge stainless steel guide cannula targeted at the $\mathrm{LH}$, the medial $\mathrm{PFH}$, the thalamus (THAL) dorsal to these sites, the paraventricular nucleus (PVN) medial to these sites, the amygdala (AMY) lateral to these sites, the anterior lateral hypothalamus (ALH), or the posterior lateral hypothalamus (PLH). For rats weighing 400-425 gm, with the incisor bar at $-3.3 \mathrm{~mm}$, coordinates (in $\mathrm{mm}$ ) were 6.3 anterior to the interaural line (A), 2.0 lateral to the midsagittal sinus (L), and 8.0 ventral to the surface of the skull (V) for the $\mathrm{LH}$; $6.3 \mathrm{~A}, 1.0 \mathrm{~L}$, and $8.0 \mathrm{~V}$ for $\mathrm{PFH}$; $6.3 \mathrm{~A}, 1.8$ $\mathrm{L}$, and $5.4 \mathrm{~V}$ for THAL; $6.8 \mathrm{~A}, 0.4 \mathrm{~L}$, and $7.4 \mathrm{~V}$ for PVN; $6.3 \mathrm{~A}, 3.5 \mathrm{~L}$, and 8.2 V for AMY; 7.7 A, 1.8 L, and 8.2 V for ALH; and 4.6 A, 1.6 L, and $7.7 \mathrm{~V}$ for PLH. For rats weighing $>425 \mathrm{gm}, 0.1 \mathrm{~mm}$ was added in the anterior direction. The cannula was permanently anchored to the skull with dental acrylic and stainless steel screws, and a plastic guard was placed around the exposed portion of the cannula. To maintain patency, a 33 gauge stainless steel obturator was inserted into the lumen of the cannula. Animals were allowed at least 1 week of postoperative recovery, during which they were repeatedly handled and mock-injected to accustom them to the testing procedure.

General test procedure. Tests were conducted during the early portion of the light phase with freshly prepared mash diet provided to each subject at least $1.5 \mathrm{hr}$ before testing to maximize satiety. Central injections were administered in a volume of $0.3 \mu$ l through a 33 gauge injector terminating in the brain $1 \mathrm{~mm}$ below the tip of the guide cannula. Food intake was measured 1,2 , and $4 \mathrm{hr}$ after the final injection. For each experiment, all treatments were administered in counterbalanced order in tests separated by at least $48 \mathrm{hr}$. Experiments were completed within 5 weeks after surgery.

Pharmacological agents. 8-br-cAMP (sodium salt) and 3-isobutyl-1methylxanthine (IBMX) were obtained from Sigma (St. Louis, MO). A water soluble analog of forskolin, 7-deacetyl-7-O-( $N$-methylpiperazino)$\gamma$-butyryl-forskolin dihydrochloride (MPB forskolin), was obtained from Calbiochem (San Diego, CA). IBMX was dissolved in dimethyl sulfoxide (DMSO). All other agents were dissolved in artificial CSF (aCSF) composed of (in mM): $\mathrm{Na}^{+} 147, \mathrm{Cl}^{-} 154, \mathrm{~K}^{+} 3, \mathrm{Ca}^{2+} 1.2$, and $\mathrm{Mg}^{2+} 0.9$, pH 7.4.

Experiment 1. To determine the locus of 8-br-cAMP feeding stimulation, the membrane-permeant cAMP analog 8-br-cAMP $(1,10$, and 100 nmol) or its aCSF vehicle was injected into the PFH $(n=8), \mathrm{LH}(n=$ $10)$, THAL $(n=6)$, PVN $(n=9)$, AMY $(n=6)$, ALH $(n=8)$, or PLH $(n=6)($ total $n=53)$.

Experiment 2. To determine the consistency in the magnitude of the observed eating responses and to better localize the effect of 8-br-cAMP, a naive group of animals $(n=33)$ implanted with PFH $(n=12), \mathrm{LH}$ $(n=11)$, or THAL $(n=10)$ cannulas was injected with $100 \mathrm{nmol}$ of 8-br-cAMP or aCSF. Data for each subject were averaged over two trials of the counterbalanced series.

Experiment 3. To examine the anatomical specificity of eating elicited by compounds that increase endogenous cellular cAMP, a separate group of animals (total $n=59$ ) received central injections of IBMX (30, 150 , or $300 \mathrm{nmol}$ ) followed $10 \mathrm{~min}$ later by a matched dose of MPB forskolin or injection of DMSO vehicle followed by aCSF vehicle. Animals with cannulas targeted at the PFH $(n=14)$, LH $(n=9)$, THAL $(n=11), \operatorname{PVN}(n=6)$, AMY $(n=6), \operatorname{ALH}(n=7)$, and PLH $(n=6)$ received the resulting four treatments in counterbalanced order. To determine whether IBMX alone or MPB forskolin alone could elicit eating, after completing this first block of tests the subjects remaining with intact headpieces and cannulas $(n=52)$ were injected in counterbalanced order with DMSO followed 10 min later by aCSF, $300 \mathrm{nmol}$ of IBMX followed by aCSF, or DMSO followed by $300 \mathrm{nmol}$ of MPB forskolin.

Histological verification of cannula placements and data analysis. On completion of testing, subjects were killed by $\mathrm{CO}_{2}$ inhalation and perfused transcardially with $10 \%$ Formalin. Brains were removed and postfixed for at least $24 \mathrm{hr}$ in Formalin before sectioning on a cryotome. Coronal sections $(100 \mu \mathrm{m})$ were cut through the extent of the visible cannula track and stained with cresyl violet. The injection sites were then localized by tracing the image onto size-matched figures adapted from the atlas of Paxinos and Watson (1986). Food intake data were averaged and analyzed for effects of drug and injection site using ANOVA, with multiple comparisons (Duncan's multiple range test) performed at an $\alpha$ of 0.05 .

\section{RESULTS}

\section{Histology}

The injection sites for 140 of the 145 subjects were localized histologically; all of these were located in their intended targets. Photomicrographs of representative injection sites within the seven brain sites tested are shown in Figure 1.

\section{Experiment 1}

Seven brain sites within or bracketing the hypothalamus were tested for sensitivity to the eating-stimulatory effect of 8-brcAMP (1-100 nmol). As shown in Figure 2, the membranepermeant $\mathrm{cAMP}$ analog stimulated eating only after injection into the PFH, LH, and THAL. Statistical analysis revealed 

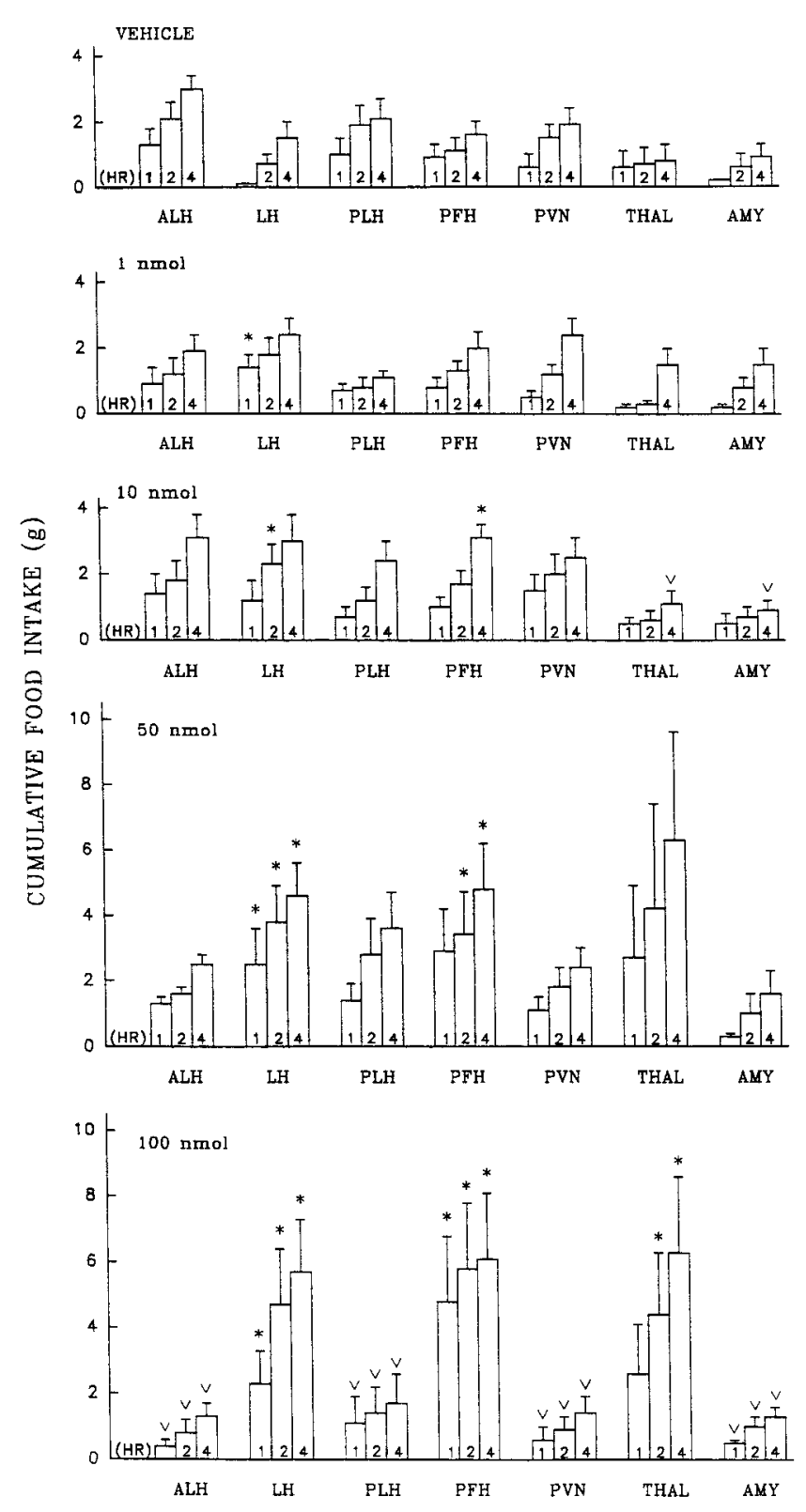

Figure 2. Cumulative food intake 1, 2, and $4 \mathrm{hr}$ after injection (mean \pm SEM) as a function of injection site $(A L H$, anterior hypothalamus; $L H$, lateral hypothalamus; $P L H$, posterior lateral hypothalamus; $P F H$, perifornical hypothalamus; $P V N$, paraventricular nucleus; $T H A L$, thalamus; $A M Y$, amygdala) and dose of $0-100 \mathrm{nmol}$ of 8-br-cAMP. ${ }^{*} p<0.05$, greater than vehicle at the corresponding time. Downward carats indicate lower than PFH and LH scores at the corresponding time; $p<0.05$, both by Duncan's multiple range test.

significant effects of both injection site and drug dose $1 \mathrm{hr}\left(F_{6,230}\right.$ $=2.71 ; p<0.02 ; F_{4,230}=3.91 ; p<0.005$, respectively), $2 \mathrm{hr}$ $\left(F_{6,230}=2.75 ; p<0.02 ; F_{4,230}=5.27 ; p<0.0005\right.$, respectively $)$, and $4 \mathrm{hr}$ after injection $\left(F_{6,230}=3.66 ; p<0.002 ; F_{4,230}=6.25\right.$; $p<0.0002)$. The only significant interaction was between drug dose and injection site at $4 \mathrm{hr}$ after injection $\left(F_{24,230}=1.85 ; p<\right.$ $0.0002)$. Multiple comparison tests showed that significant eating was elicited at some postinjection times after injection of 8-brcAMP into the LH with doses as low as $1 \mathrm{nmol}$ and the PFH with doses as low as $10 \mathrm{nmol}(p<0.05)$, and reliable eating was obtained with injections of 50 and $100 \mathrm{nmol}$ of 8-br-cAMP in both of these sites. The $50 \mathrm{nmol}$ dose elicited eating of $3.4 \mathrm{gm}$ in $2 \mathrm{hr}$, and the highest dose of 8-br-cAMP (100 nmol) elicited food intake of $4.7 \pm 1.7$ and $5.8 \pm 2.0 \mathrm{gm}$ in $2 \mathrm{hr}$ in the $\mathrm{LH}$ and $\mathrm{PFH}$, respectively. Intake $4 \mathrm{hr}$ after injection into the $\mathrm{LH}$ and $\mathrm{PFH}$ was $5.7 \pm 1.6$ and $6.1 \pm 2.0 \mathrm{gm}$, respectively. Although there was no significant increase in eating during the first hour, injection of the highest dose of 8-br-cAMP into the THAL dorsal to the $\mathrm{LH}$ and PFH elicited significant eating $2 \mathrm{hr}(4.4 \pm 1.9 \mathrm{gm})$ and $4 \mathrm{hr}$ after injection $(6.3 \pm 2.3 \mathrm{gm})(p<0.05)$. The eating response to 8 -br-cAMP injected into the THAL was more variable between individuals and of a longer latency than the response obtained after injection into the $\mathrm{LH}$ or $\mathrm{PFH}$, suggesting that $\mathrm{LH}$ and $\mathrm{PFH}$ injections caused eating via actions of 8-br-cAMP on local brain tissue rather than after diffusion dorsally to the THAL. Injection of 8-br-cAMP into areas bracketing the $\mathrm{PFH}$ and $\mathrm{LH}$ in all other directions was ineffective in stimulating eating.

Figure $3 A$ presents food intake induced by 8 -br-cAMP as a function of the mean histologically derived injection sites for all brain areas tested. As shown here, the LH and PFH injection sites were bracketed dorsally by the THAL, medially by the PVN, laterally by the AMY, anteriorly by the ALH, and posteriorly by the PLH. The PFH, LH, THAL, and AMY injections were located at approximately the same coronal level: lateral to and just posterior to the PVN. The PFH and $\mathrm{LH}$ injections were separated by an average of $1 \mathrm{~mm}$. Injection sites in the AMY were within the medial nucleus, the basomedial nucleus, and the anterior cortical nucleus. The PVN injections were located an average of $0.8 \mathrm{~mm}$ from the PFH injection sites. ALH injections were placed an average of $1.1 \mathrm{~mm}$ anterior to the $\mathrm{LH}$, and the PLH injections were placed an average of $2 \mathrm{~mm}$ posterior to the $\mathrm{LH}$. The THAL injections were placed an average of $1.9 \mathrm{~mm}$ dorsal to the $\mathrm{LH}$ in the ventrolateral, ventromedial, and ventral posterolateral thalamic nuclei. As shown in Figure $3 A$, injection of 100 nmol of 8-br-cAMP elicited significant eating within the first hour after injection into the $\mathrm{LH}$ and $\mathrm{PFH}$, whereas all other sites, including the THAL dorsal to the $\mathrm{LH}$ and the PVN just $0.8 \mathrm{~mm}$ medial to the PFH, injections were ineffective.

\section{Experiment 2}

As shown in Figure 4 and consistent with the previous results, in a group of naive animals $100 \mathrm{nmol}$ of 8 -br-cAMP elicited marked eating after injection into the $\mathrm{LH}$ and $\mathrm{PFH}$ and elicited a smaller eating response after injection into the THAL. Statistical analysis revealed significant effects of both drug treatment and injection site at 1 and $2 \mathrm{hr}$ after injection $\left(F_{1,60}=42.88 ; p<0.0001 ; F_{2,60}\right.$ $=3.67 ; p<0.04$ at $1 \mathrm{hr} ; F_{1,60}=47.25 ; p<0.0001 ; F_{2,60}=4.10$; $p<0.03$ at 2 hr), with 8-br-cAMP eliciting significant feeding after injection into all three sites tested. By $4 \mathrm{hr}$ after injection, the effect of drug treatment only was significant $\left(F_{1,60}=35.94\right.$; $p<0.0001$ ), and $4 \mathrm{hr}$ eating scores after 8-br-cAMP were significantly greater than vehicle scores in all three sites tested. Although 8-br-cAMP was effective in stimulating eating when injected into the LH, PFH, and THAL, injections into the THAL resulted in a significantly smaller feeding response within the $2 \mathrm{hr}$ after injection than that elicited by $\mathrm{LH}$ or PFH injections $(p<$ $0.05)$. Food intake $2 \mathrm{hr}$ after injection into the THAL $(4.0 \pm 1.0$ gm) was $<48 \%$ of that observed after injection into the $\mathrm{PFH}$ $(8.6 \pm 1.2 \mathrm{gm})$ and $\mathrm{LH}(8.5 \pm 1.8 \mathrm{gm})$.

\section{Experiment 3}

As shown in Figure 5, eating was significantly stimulated by combined injection of the phosphodiesterase inhibitor IBMX and 
A

$$
8-\mathrm{br}-\mathrm{cAMP}
$$

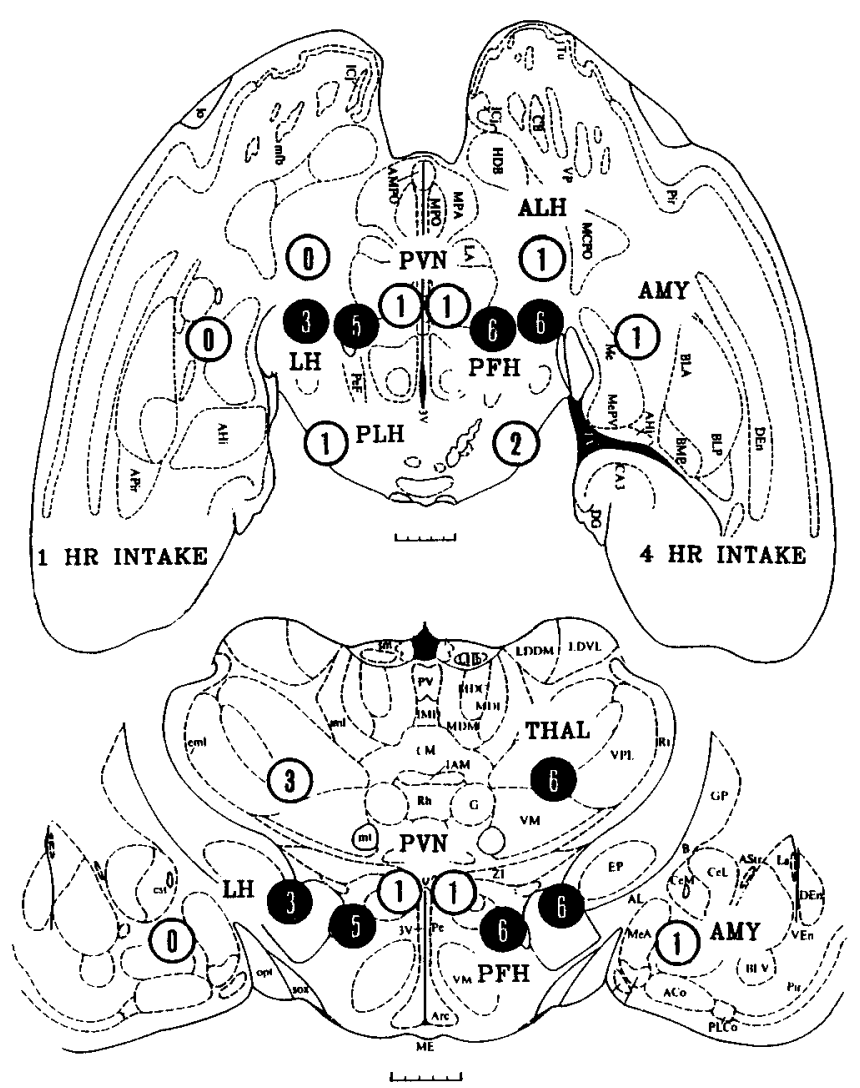

B

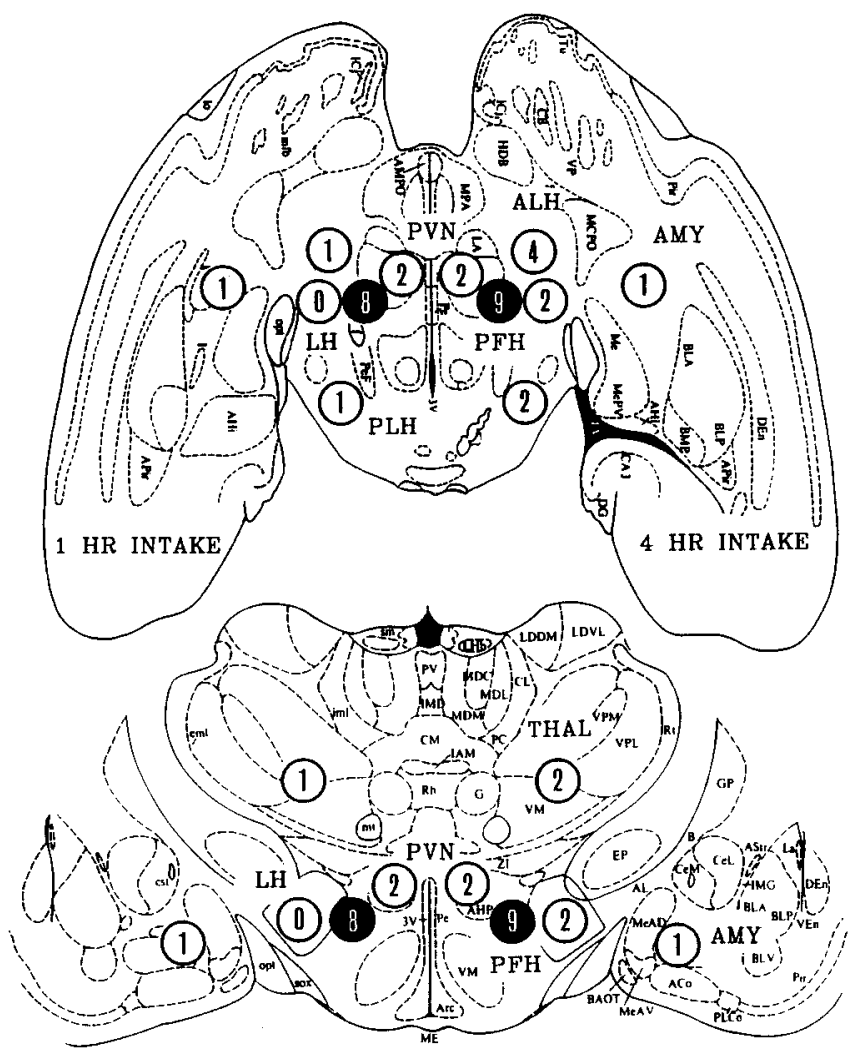

Figure 3. Brain sites from which eating is elicited by injection of 8-br-cAMP and agents that increase endogenous cAMP. Cumulative food intake, rounded to the nearest gram, elicited by $100 \mathrm{nmol}$ of 8-br-cAMP $(A)$ or combined $300 \mathrm{nmol}$ of IBMX and MPB forskolin $(B), 1$ (left side of brain) and $4 \mathrm{hr}$ (right side of brain) after injection into the histologically determined mean sites represented by the circles (filled circles represent sites from which eating was elicited; $p<0.05$, Duncan's multiple range test; open circles represent ineffective sites) on size-matched schematics (Paxinos and Watson, 1986) in the horizontal (top) and coronal (bottom) planes. In the top schematics, sections in the horizontal plane have been collapsed to depict the anteroposterior and mediolateral relation of the injection sites to each other, whereas in the bottom planes, anteroposterior sections have been collapsed to depict the dorsoventral and mediolateral position of the injection sites. $A L H$, Anterior hypothalamus; $L H$, lateral hypothalamus; $P L H$, posterior lateral hypothalamus; $P F H$, perifornical hypothalamus; $P V N$, paraventricular nucleus; $T H A L$, thalamus; $A M Y$, amygdala.

the adenylyl cyclase activator MPB forskolin only into the PFH. A significant interaction between injection site and drug dose was found at $1 \mathrm{hr}\left(\mathrm{F}_{18,208}=4.54 ; p<0.0001\right), 2 \mathrm{hr}\left(\mathrm{F}_{18,208}=3.28 ; p<\right.$ $0.0001)$, and $4 \mathrm{hr}\left(\mathrm{F}_{18,208}=2.43 ; p<0.005\right)$ after injection. A significant effect of drug dose alone on eating was found only in the $\operatorname{PFH}\left(\mathrm{F}_{3,52}=11.61 ; p<0.0001\right.$ at $1 \mathrm{hr}$ after injection; $\mathrm{F}_{3,52}=$ 9.75; $p<0.001$ at $2 \mathrm{hr}$ after injection; and $\mathrm{F}_{3,52}=3.73 ; p<0.02$ at $4 \mathrm{hr}$ after injection), with stimulation of eating compared with vehicle scores occurring after combined injection of the highest doses (300 nmol) of IBMX and MPB forskolin $(p<0.05$ at all time points). The eating response was robust, with food intake 2 hr after injection of this dose into the PFH averaging $8.4 \pm 2.0$ gm, compared with an average intake of $1.3 \pm 0.3 \mathrm{gm} 2 \mathrm{hr}$ after injection of vehicle solution. In contrast, combined injection of IBMX and MPB forskolin into all other surrounding brain sites failed to stimulate eating with respect to vehicle scores.

A significant effect of injection site alone on eating was found only at the effective dose (300 nmol) of IBMX and MPB forskolin $\left(\mathrm{F}_{6,52}=5.74 ; p<0.0001\right.$ at $1 \mathrm{hr}$ after injection; $\mathrm{F}_{6,52}=4.81 ; p<$ 0.0006 at $2 \mathrm{hr}$ after injection; and $\mathrm{F}_{6,52}=4.36 ; p<0.0012$ at $4 \mathrm{hr}$ after injection), with eating after injection into the $\mathrm{PFH}$ being significantly greater than that resulting from injection into all other sites ( $p<0.05$ by Duncan's multiple range test at all time points).

Figure $3 B$ depicts the histologically determined mean injection sites for all brain areas examined and shows that the effective $\mathrm{PFH}$ injections were bracketed in all directions by ineffective sites. The distribution of tested brain sites is similar to that of Experiment 1. The effective PFH injections were concentrated at a coronal level $\sim 0.5 \mathrm{~mm}$ posterior to the ineffective PVN injections and $0.7 \mathrm{~mm}$ medial to the average LH placement. The ALH injections $0.9 \mathrm{~mm}$ anterior to the $\mathrm{LH}$ and the PLH injections 1.6 $\mathrm{mm}$ posterior to the $\mathrm{LH}$ were also ineffective in stimulating eating. Likewise, injections into the THAL $\sim 1.6 \mathrm{~mm}$ dorsal to the $\mathrm{LH}$ at the same coronal level as the $\mathrm{PFH}$ and $\mathrm{LH}$ failed to stimulate eating, as did injections into the AMY nuclei slightly anterior to and $\sim 2.3 \mathrm{~mm}$ lateral to the PFH. Eating elicited by combined injection of IBMX and MPB forskolin (300 nmol) into the $\mathrm{PFH}$ was $8.1 \pm 2.0$ and $8.8 \pm 2.0 \mathrm{gm}$ at 1 and $4 \mathrm{hr}$ after injection, respectively, whereas injection of this dose into sites other than the PFH never exceeded a mean of $3.8 \mathrm{gm}$ at any time point measured. 


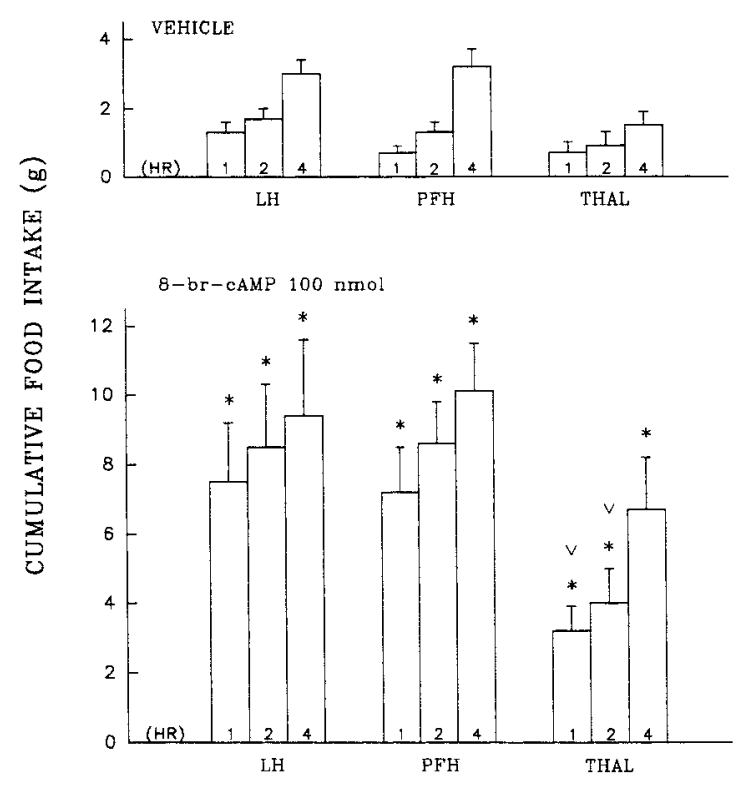

Figure 4. Cumulative food intake 1, 2, and $4 \mathrm{hr}$ after injection (mean \pm SEM) elicited by $100 \mathrm{nmol}$ of 8-br-cAMP as a function of injection site ( $L H$, lateral hypothalamus; $P F H$, perifornical hypothalamus; $T H A L$, thalamus). ${ }^{*} p<0.05$, greater than vehicle at the corresponding time; downward carats indicate lower than PFH and LH scores at the corresponding time; $p<0.05$, both by Duncan's multiple range test.

In contrast to the effectiveness of combined injection of IBMX and MPB forskolin (300 nmol) in the PFH, neither compound alone stimulated eating in any brain site tested $(p>0.20$ by ANOVA) (data not shown).

\section{DISCUSSION}

The present study demonstrates that functionally increasing cAMP concentrations by means of 8-br-cAMP (Greengard, 1978) injection into the $\mathrm{PFH}$ and $\mathrm{LH}$ elicits eating, whereas injections into other sites are either less effective or ineffective. The effective sites in the PFH and LH were bracketed anteriorly, posteriorly, medially, and laterally at average distances of only $0.8-2.0$ $\mathrm{mm}$ by ineffective sites, and the only other brain site into which injection of $100 \mathrm{nmol}$ of 8 -br-cAMP stimulated eating was the THAL $1.9 \mathrm{~mm}$ dorsal to the LH. One question is to what extent 8-br-cAMP injected into the PFH and LH stimulated feeding by a local action in these sites, or by diffusion to dorsal brain structures, such as the THAL. Eating consequent to diffusion from the LH and PFH seems unlikely, however, because THAL injections were effective only at the highest dose of 8-br-cAMP tested $(100 \mathrm{nmol})$, and even this dose did not consistently stimulate eating within the first hour after injection into the THAL, in contrast to the consistent stimulation at this time after PFH and LH injections (Figs. 2, 4). When eating was elicited during the first hour after THAL injections, 1 and $2 \mathrm{hr}$ food intake was $<48 \%$ of the food intake of PFH- and LH-injected subjects (Fig. 4). Additionally, the eating elicited by THAL injections might be mediated by biochemical mechanisms distinct from those that produce eating after $\mathrm{PFH}$ and $\mathrm{LH}$ injections. This was suggested by our previous evidence that the cGMP analog 8-br-cGMP was completely ineffective in eliciting eating after injection into the $\mathrm{PFH}$ or LH but did elicit a feeding response after THAL injection that was equivalent to that elicited by 8-br-cAMP injected into that site (Gillard et al., 1997a). Further supporting local, rather than thalamic, actions of $\mathrm{PFH}$ injections is the current
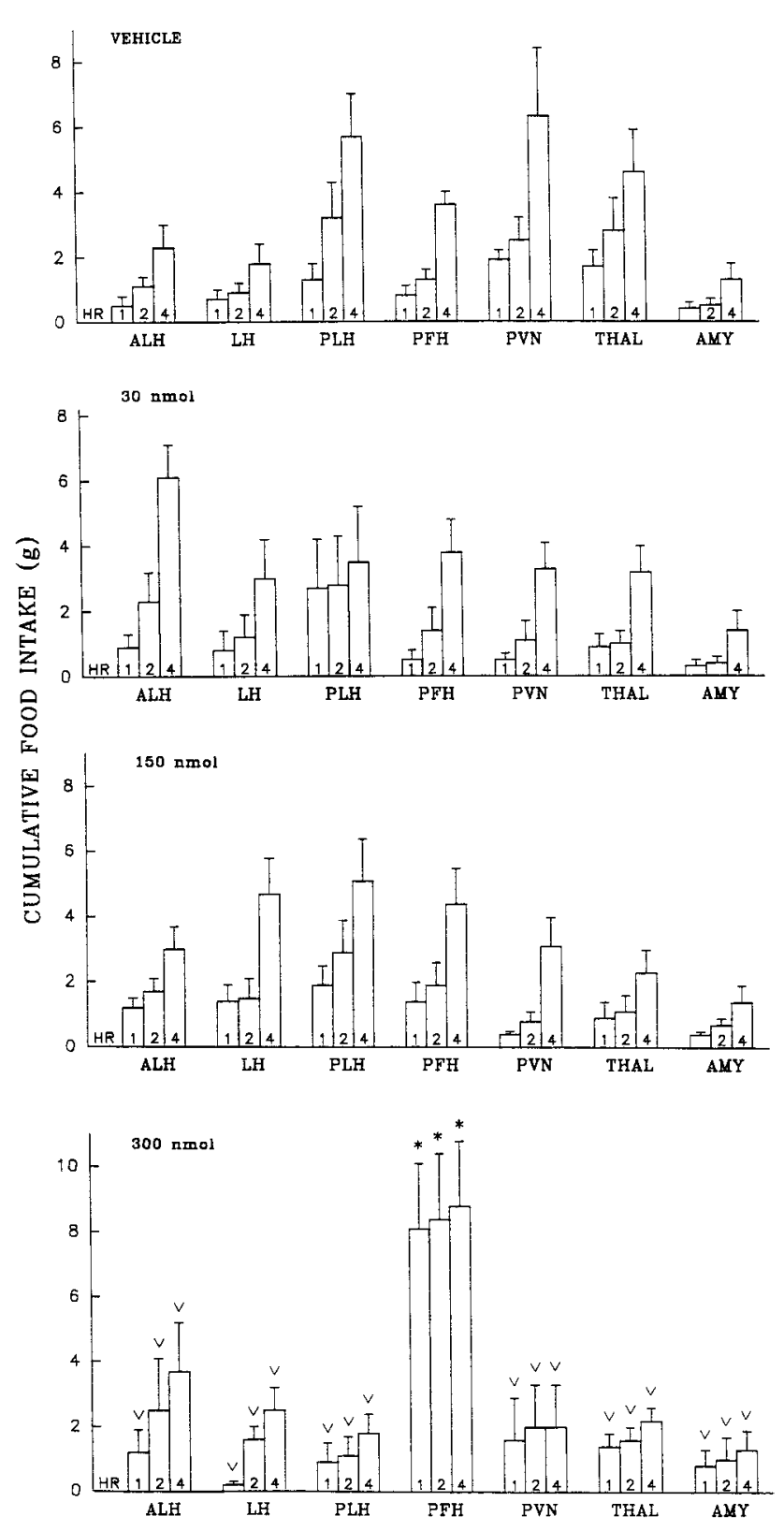

Figure 5. Cumulative food intake 1, 2, and $4 \mathrm{hr}$ after injection (mean \pm SEM) as a function of injection site $(A L H$, anterior hypothalamus; $L H$, lateral hypothalamus; $P L H$, posterior lateral hypothalamus; $P F H$, perifornical hypothalamus; $P V N$, paraventricular nucleus; $T H A L$, thalamus; $A M Y$, amygdala) and matched doses of 0-300 nmol of IBMX and MPB forskolin. ${ }^{*} p<0.05$, greater than vehicle at the corresponding time; downward carats indicate lower than $\mathrm{PFH}$ scores at the corresponding time; $p<0.05$, both by Duncan's multiple range test.

finding that agents that increase endogenous cAMP (IBMX and MPB forskolin) stimulated eating after injection into the PFH but not the THAL. Although diffusion and reflux of centrally injected chemicals up the cannula track into the cerebroventricles can occur and may mediate behavioral effects (Myers et al., 1971; Johnson and Epstein, 1975), the smaller effects observed with THAL injections close to the lateral ventricles and the ineffectiveness of injections into the PVN just lateral to the third ventricle argue against mediation of eating by diffusion or reflux of 8-br-cAMP into the ventricular system. Collectively, these data argue that 8-br-cAMP elicited feeding after injection into the LH 
and $\mathrm{PFH}$ via an action on a cellular cAMP effector in these locations rather than after diff usion or reflux to the THAL or the cerebroventricles, because if the 8-br-cAMP was acting after diffusion to these sites, then the THAL injections would be expected to yield greater responses than $\mathrm{LH}$ and $\mathrm{PFH}$ injections.

It is ultimately of interest to determine whether the eating observed after introduction of exogenous 8-br-cAMP reflects cellular processes within the PFH that are relevant to the physiological control of food intake. The present study confirms that injection of drugs that increase endogenous cAMP can stimulate intense eating (Gillard et al., 1997a), and it is the first to show that this effect is attributable to actions specifically in the PFH. We have shown previously that $100 \mathrm{nmol}$ of cAMP itself, which does not cross cell membranes appreciably, does not stimulate eating in the PFH and LH (Gillard et al., 1997a), suggesting that the membrane-permeant cAMP analog 8-br-cAMP stimulates eating via an intracellular action on local cell bodies or processes rather than via osmotic effects or activation of adenosine receptors. Consistent with this, we demonstrated in the present study that combined PFH injection of the phosphodiesterase inhibitor IBMX, which reduces the breakdown of cAMP, and the AC stimulator MPB forskolin, which stimulates cAMP production, significantly stimulates eating of up to $8.8 \pm 2.0$ gm (Fig. 5). Several findings argue that combined IBMX-MPB forskolin injection elicited eating via actions on a common system, the cAMP system. Although IBMX can have phosphodiesteraseindependent effects (Schwabe et al., 1978), and unmodified forskolin is known to have cAMP-independent effects (McHugh and McGee, 1986; Laurenza et al., 1989; Baxter and Byrne, 1990), the failure of either compound alone to elicit eating when injected unilaterally argues against mediation of eating by the different nonspecific effects of either compound. In addition, preliminary studies have shown that whereas bilateral PFH injection of 300 nmol of MPB forskolin alone elicited eating, a forskolin analog having the same non-cAMP-dependent effects as forskolin but lacking cAMP-related activity (Laurenza et al., 1989) did not (Gillard et al., 1997b). These results collectively argue against mediation of eating by either osmotic effects or non-cAMPdependent effects and, when combined with the ineffectiveness of IBMX-MPB forskolin in the other sites tested (particularly the LH and THAL), argue for the PFH as the most likely locus of feeding stimulation by endogenous intracellular cAMP.

Several findings also suggest that the effects of 8-br-cAMP and agents that increase endogenous cAMP are attributable to actions in the PFH rather than in the nearby ventromedial hypothalamic nucleus (VMH), which has also been implicated in eating control. Specifically, in contrast to the effectiveness of $\mathrm{PFH}$ injections of 8-br-cAMP and agents that increase cAMP, injections into the $\mathrm{PVN} \sim 0.8 \mathrm{~mm}$ medial to the $\mathrm{PFH}$ were ineffective despite the proximity of these injections to the $\mathrm{VMH}$, also located medially to the PFH. In addition, separate experiments in our laboratory have shown that VMH injections of 8-br-cAMP and IBMX-MPB forskolin at the same doses used in the present study do not elicit significant increases in eating with respect to vehicle injections $(p>0.2)$ (E. R. Gillard, A. M. Khan, B. G. Stanley, unpublished observations).

Why did application of agents that typically increase endogenous CAMP elicit eating with much greater anatomical specificity than did microinjection of 8-br-cAMP? The disparity may simply reflect differences in the pattern and extent of diffusion in brain tissue among these chemically diverse compounds. However, the major functional difference among these compounds is their mode of action. Whereas 8-br-cAMP penetrates cells indiscriminately, the ability of both IBMX and MPB forskolin to increase cAMP should be dependent on cellular phosphodiesterase and adenylyl cyclase, with IBMX-MPB forskolin treatment being most effective in cells having the highest levels of phosphodiesterase and adenylyl cyclase. Consistent with this idea is evidence that the abundance and types of adenylyl cyclase vary markedly across different brain sites (Williams et al., 1969; Worley et al., 1986; Xia et al., 1992; Mons et al., 1993; Cali et al., 1994; Mons and Cooper, 1994). Such a difference in sensitivity between cells in different areas, coupled with the requirement for relatively high doses to be effective in stimulating eating, might account for the finding that IBMX-MPB forskolin elicited eating only when applied in the PFH and not in the $\mathrm{LH}$, although neurons in both of these areas have demonstrated roles in orchestrating eating behavior, and both are responsive to the eating-stimulatory effect of 8-br-cAMP.

Although further study is required to resolve the identity of the cellular elements on which 8-br-cAMP and IBMX-MPB forskolin act in the $\mathrm{PFH}$, that injections into other sites were ineffective suggests that stimulation of cell bodies or processes in the PFH rather than of fibers of passage elicited eating. Injections into areas bracketing the $\mathrm{PFH}$ and $\mathrm{LH}$, particularly the ALH and PLH, would be expected to intersect the course of major fiber systems coursing through the LH and PFH. It is unknown which cell types in the PFH are affected by local increases in cAMP resulting from application of 8-br-cAMP or agents that increase endogenous cAMP. However, the abundance of evidence implicating PFH neurons in natural eating, as well as the particular effectiveness of PFH electrical stimulation in eliciting eating (Leibowitz and Stanley, 1986; Murzi et al., 1991), suggests that these agents may act on neurons intrinsic to this area and/or on the local processes of extrinsic neurons to stimulate eating.

The results of the present study suggest that cAMP may be an important second messenger in PFH neurons participating in a neural circuit concerned specifically with eating regulation. In this regard, it is interesting that the hypothalamus has a high rate of cAMP production (Williams et al., 1969). Because its activity is subject to regulation by inhibitory and stimulatory G-proteins as well as components of other signaling systems, the cAMPsynthesizing enzyme AC has the potential to act as a coincidence detector for the activation of a variety of metabotropic neurotransmitter receptors (Bourne and Nicoll, 1993; Lustig et al., 1993). The cAMP second messenger system may be a powerful means of integrating the actions of multiple neurotransmitters that affect eating through actions in the PFH. Although it is unknown which neurotransmitters might act directly or indirectly on the cAMP system to stimulate food intake, the PFH is most sensitive to the eating-suppressive effects of dopamine and epinephrine and the eating-stimulatory effect of neuropeptide $Y$ (NPY) (Stanley et al., 1993), suggesting that PFH cAMP may contribute to the effects of these neurochemicals. However, all of the known NPY receptor subtypes couple to decreases in cAMP, suggesting that increased PFH cAMP is unlikely to elicit eating by acting exclusively on PFH cells directly responsive to NPY. Rather, increases in cAMP may act on a variety of cell types, with the effects on neurons downstream of NPY-responsive neurons being critical for eating stimulation. That PFH NPY may ultimately induce increases in cAMP in neurons downstream of those bearing NPY receptors is consistent with recent findings that both PFH injection of NPY and food deprivation increased cAMP response element binding to rat hypothalamic nuclear 
extracts (Sheriff et al., 1997). Although this explanation appears to be the most parsimonious, it may be premature to dismiss the possibility that increased cAMP within NPY-responsive neurons might contribute to the observed stimulation of eating, given that it has not been clearly established that NPY elicits eating exclusively by decreasing cAMP in the eating-relevant neurons bearing NPY receptors. Specifically, (1) eating-relevant NPY receptors may couple to other second messenger systems, such as increases in intracellular $\mathrm{Ca}^{2+}$ (Herzog et al., 1992); (2) pertussis toxin, which blocks both eating and inhibition of cAMP production in response to NPY (Chance et al., 1989), also blocks increases in intracellular $\mathrm{Ca}^{2+}$ mediated by the $\mathrm{G}_{\mathrm{o}}$ protein; and (3) PFH NPY has been shown to result in increased activity of hypothalamic calcium- and calmodulin-dependent protein kinase II (Sheriff et al., 1997). These findings may suggest that the effects of PFH NPY and cAMP on eating might not be mutually exclusive. Consistent with this, our preliminary studies indicate that $\mathrm{PFH}$ administration of 8-br-cAMP does not reduce eating elicited by NPY (E. R. Gillard, A. M. Khan, A. U. Haq, B. G. Stanley, unpublished observations).

The dramatic stimulation of eating by increases in PFH cAMP suggests that this second messenger may play a role in a neural circuit(s) controlling eating. In addition to integrating the effects of multiple neurotransmitters in the PFH, cAMP may also contribute to the plasticity evidenced by PFH neurons during foodrelated learning (Mora et al., 1976).

\section{REFERENCES}

Anand BK, Brobeck JR (1951) Hypothalamic control of food intake in rats and cats. Yale J Biol Med 24:123-140.

Baxter DA, Byrne JH (1990) Reduction of voltage-activated $\mathrm{K}^{+}$currents by forskolin is not mediated via cAMP in pleural sensory neurons of Aplysia. J Neurophysiol 64:1474-1483.

Bourne HR, Nicoll R (1993) Molecular machines integrate coincident synaptic signals. Cell [Suppl] 72:65-75.

Cali JJ, Zwaagstra JC, Mons N, Cooper DMF, Krupinski J (1994) Type VIII adenylyl cyclase. J Biol Chem 269:12190-12195.

Chance WT, Sheriff S, Foley-Nelson T, Fischer JE, Balasubramaniam A (1989) Pertussis toxin inhibits neuropeptide Y-induced feeding in rats. Peptides 10:1283-1286.

Cheng J-T, Sessler FM, Azizi SA, Chapin JK, Waterhouse B (1988) Electrophysiological actions of norepinephrine in rat lateral hypothalamus. II. An in vitro study of the effects of iontophoretically applied norepinephrine on $\mathrm{LH}$ neuronal responses to $\gamma$-aminobutyric acid (GABA). Brain Res 446:90-105.

Gillard ER, Khan AM, Haq AU, Grewal RS, Stanley BG (1994) Stimulation of feeding behavior in the rat by intrahypothalamic injection of 8-br-cAMP. Soc Neurosci Abstr 20:1680.

Gillard ER, Khan AM, Haq AU, Grewal RS, Mouradi B, Stanley BG (1997a) Stimulation of eating by the second messenger cAMP in the perifornical and lateral hypothalamus. Am J Physiol 273:R107-R112.

Gillard ER, Mouradi B, Khan AM, Wolfsohn S, Stanley BG (1997b) Eating elicited by elevation of endogenous cAMP in the perifornical hypothalamus $(\mathrm{PFH})$ of the rat is anatomically specific. Soc Neurosci Abstr 23:577.

Greengard P (1978) Cyclic nucleotides, phosphorylated proteins, and neuronal function. New York: Raven.

Herzog H, Hort YJ, Ball HJ, Hayes G, Shine J, Selbie LA (1992) Cloned human neuropeptide Y receptor couples to two different second messenger systems. Proc Natl Acad Sci USA 89:5794-5798.
Himmi T, Boyer A, Orsini JC (1988) Changes in lateral hypothalamic neuronal activity accompanying hyper- and hypoglycemias. Physiol Behav 44:347-354.

Johnson AK, Epstein AN (1975) The cerebral ventricles as the avenue for the dipsogenic action of intracranial angiotensin. Brain Res $86: 399-418$.

Laurenza A, Sutkowski EM, Seamon KB (1989) Forskolin: a specific stimulator of adenylyl cyclase or a diterpene with multiple sites of action? Trends Pharmacol Sci 10:442-447.

Leibowitz SF, Stanley BG (1986) Neurochemical controls of appetite. In: Feeding behavior: neural and humoral controls (Ritter RC, Ritter S, Barnes CD, eds), pp 191-234. Orlando, FL: Academic.

Lustig KD, Conklin BR, Herzmark P, Taussig R, Bourne HR (1993) Type II adenylylcyclase integrates coincident signals from $G_{s}, G_{i}$, and $\mathrm{G}_{\mathrm{q}}$. J Biol Chem 268:13900-13905.

McHugh EM, McGee Jr R (1986) Direct anesthetic-like effects of forskolin on the nicotinic acetylcholine receptors of PC12 cells. J Biol Chem 261:3103-3106.

Mons N, Yoshimura M, Cooper DMF (1993) Discrete expression of $\mathrm{Ca}^{2+} /$ calmodulin-sensitive and $\mathrm{Ca}^{2+}$-insensitive adenylyl cyclases in the rat brain. Synapse 14:51-59.

Mons N, Cooper DMF (1994) Selective expression of one $\mathrm{Ca}^{2+}$ inhibitable adenylyl cyclase in dopaminergically innervated rat brain regions. Mol Brain Res 22:236-244.

Mora F, Rolls ET, Burton MJ (1976) Modulation during learning of the responses of neurons in the lateral hypothalamus to the sight of food. Exp Neurol 53:508-519.

Murzi E, Baptista T, Hernandez L (1991) Hypothalamic sites affecting masticatory neurons in rats. Brain Res Bull 26:321-325.

Myers RD, Tytell M, Kawa A, Rudy T (1971) Micro-injection of ${ }^{3} \mathrm{H}$ acetylcholine, ${ }^{14} \mathrm{C}$-serotonin and ${ }^{3} \mathrm{H}$-norepinephrine into the hypothalamus of the rat: diffusion into tissue and ventricles. Physiol Behav 7:743-751.

Paxinos G, Watson C (1986) The rat brain in stereotaxic coordinates. Orlando, FL: Academic.

Rolls ET, Murzi E, Yaxley S, Thorpe SJ, Simpson SJ (1986) Sensoryspecific satiety: food-specific reduction in responsiveness of ventral forebrain neurons after feeding in the monkey. Brain Res 368:79-86.

Schwabe U, Ohga Y, Daly JW (1978) The role of calcium in the regulation of cyclic nucleotide levels in brain slices of rat and guinea pig. Naunyn Schmiedebergs Arch Pharmacol 302:141-151.

Sheriff S, Chance WT, Fischer JE, Balasubramaniam A (1997) Neuropeptide $\mathrm{Y}$ treatment and food deprivation increase cyclic AMP response element-binding in rat hypothalamus. Mol Pharmacol 51:597-604

Stanley BG, Magdalin W, Seirafi A, Thomas W, Leibowitz SF (1993) The perifornical area: the major focus of (a) patchily distributed hypothalamic neuropeptide $\mathrm{Y}$ sensitive feeding system(s). Brain Res 604:304-317.

Stanley BG, Willett III VL, Donias HW, Dee II MG, Duva MA (1996) Lateral hypothalamic NMDA receptors and glutamate as physiological mediators of eating and weight control. Am J Physiol 270:R443-R449.

Williams RH, Little SA, Ensinck JW (1969) Adenyl cyclase and phosphodiesterase activities in brain areas of man, monkey, and rat. Am J Med Sci 258:190-202.

Winn P, Tarbuck A, Dunnett SB (1984) Ibotenic acid lesions of the lateral hypothalamus: comparison with the electrolytic lesion syndrome. Neuroscience 12:225-240.

Worley PF, Baraban JM, De Souza EB, Snyder SH (1986) Mapping second messenger systems in the brain: differential localizations of adenylate cyclase and protein kinase C. Proc Natl Acad Sci USA 83:4053-4057.

Xia Z, Choi E-J, Wang F, Storm DR (1992) The type III calcium/ calmodulin-sensitive adenylyl cyclase is not specific to olfactory sensory neurons. Neurosci Lett 144:169-173. 